Supporting Information

\title{
Reprogramming acetogenic bacteria with CRISPR-targeted base editing via deamination
}

Peng-Fei Xia ${ }^{1, *}$, Isabella Casini ${ }^{1}$, Sarah Schulz ${ }^{1}$, Christian-Marco Klask ${ }^{1}$, Largus T. Angenent ${ }^{1,2}$, and Bastian Molitor ${ }^{1}$

${ }^{1}$ Environmental Biotechnology Group, Center for Applied Geosciences, University of Tübingen, 72074 Tübingen, Germany

${ }^{2}$ AG Angenent, Max Planck Institute for Developmental Biology, 72076 Tübingen, Germany

${ }^{*}$ Correspondence

Peng-Fei Xia, Center for Applied Geosciences, University of Tübingen, 72074

Tübingen, Germany. E-mail: pengfei.xia@mnf.uni-tuebingen.de 
Table S1 gRNA and protospacer adjacent motifs (PAM) sequences used in this study

\begin{tabular}{lllll}
\hline gRNA & Targets $^{\text {a }}$ & Strand $^{\mathbf{b}}$ & PAM & gRNA sequence \\
\hline gRNA01 & pta & N & AGG & TTACCTTTTCATTCCCTACA \\
gRNA02 & pta & C & AGG & AAAAATTTGGAGTAAGGCAA \\
gRNA05 & pta & C & AGG & CAAATAGTAAAGACAGCTCC \\
gRNA06 & pta & N & TGG & AATTGATCACTATCTGGGCA \\
gRNA07 & pta & C & TGG & TGAAATTGATGGAAAAAATT \\
gRNA10 & adhE1 & N & AGG & ATCCATCCTATAATTCCTTC \\
gRNA11 & adhE2 & C & AGG & AACAAGTGGATGAAATTTTC \\
gRNA13 & aor1 & C & TGG & GGTCAGGGAATGCCAACTTA \\
gRNA14 & aor2 & C & TGG & GATCAAGCAGATAAGATCAG \\
gRNA15 & pta & C & AGG & TTCAAATAGTAAAGACAGCTCC \\
gRNA19 & aor1 & C & TGG & AATCAAGCAGATAAAATAAG \\
\hline
\end{tabular}

apta (CLJU_c12770), adhE1 (CLJU_c16510), adhE2 (CLJU_c16520), aor1 (CLJU_c20110), and aor2 (CLJU_c20210).

${ }^{\mathrm{b}} \mathrm{N}$ indicates non-coding strand, and $\mathrm{C}$ indicates coding strand. 
Table S2 Summary of base editing in pta using gRNA01

\begin{tabular}{ccccc}
\hline No. & $\begin{array}{c}\text { Screened } \\
\text { colonies }\end{array}$ & $\begin{array}{c}\text { Edited } \\
\text { colonies }\end{array}$ & Efficiency & Editing sita $^{\mathbf{a}}$ \\
\hline 1 & 8 & 8 & $100 \%$ & $-16(6) ;-16$ and $-17(2)$ \\
2 & 8 & 4 & $50 \%$ & $-16(3) ;-16$ and $-17(1)$ \\
3 & 16 & 9 & $56 \%$ & $-16(2) ;-16$ and $-17(5) ;-16$ and $-11(1)$ \\
4 & 11 & 4 & $36 \%$ & $-16(2) ;-16$ and $-17(2)$ \\
5 & 2 & 2 & $100 \%$ & -16 and $-17(1) ;-2,-16$ and $-17(1)$ \\
Total & 45 & $27(25)^{\mathbf{b}}$ & $60 \%(55 \%)^{c}$ & \\
\hline
\end{tabular}

${ }^{a}$ Negative numbers indicate the editing sites, and numbers in brackets indicate the number of colonies with the corresponding edit. Two colonies with mixed signals are included in the numbers here, but not in the numbers in the main text.

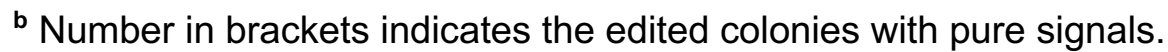

${ }^{\mathrm{c}}$ Number in brackets indicates the editing efficiency calculated with clean edited colonies only. 
Table S3 Summary of the in-silico evaluation of our base-editing tool

\begin{tabular}{lllll}
\hline \multirow{2}{*}{ Editing Type } & \multicolumn{2}{l}{ Editing window (-11 to -19) } & \multicolumn{2}{l}{ Editing window (-16 to -19) } \\
\cline { 2 - 5 } & Editable sites & Editable genes & Editable sites & Editable genes \\
\hline Missense mutation & 190568 & 4171 & 98162 & 4159 \\
Silent mutation & 66545 & 4144 & 22393 & 3896 \\
Nonsense mutation & 12745 & 3404 & 6149 & 2657 \\
Nonsense mutation $(70 \%)^{\mathbf{a}}$ & - & 3009 & - & 2203 \\
Non-editable & - & 7 & - & 21 \\
\hline
\end{tabular}

a Nonsense mutation (70\%) indicates that a premature STOP codon can be installed within the first $70 \%$ of the coding sequence of a target gene.

${ }^{b}$ Details for non-editable genes can be found in Dataset. 
Table S4 Clostridium ljungdahlii strains used in this study

\begin{tabular}{lll}
\hline Strains & Description & References \\
\hline DSM13528 & Clostridium ljungdahlii type strain & DSM13528 \\
QX3 & DSM13528, adhE1 Trp169* & This study \\
QX4 & DSM13528, adhE2 Gln33* & This study \\
QX5 & DSM13528, aor1 Gln267* & This study \\
QX6 & DSM13528, aor2 Gln267* & This study \\
\hline
\end{tabular}


Table S5 Plasmids used in this study

\begin{tabular}{|c|c|c|}
\hline Name & Description & References \\
\hline pANA1 & p15A, bla, Ф3tl methyltransferase & 1 \\
\hline pMTLdSpCas9 & pMTL82254, $\mathrm{P}_{2 \text { tet01:dcas9 }}$ & 2 \\
\hline pTargetF & ColE1, smR & 3 \\
\hline pScl_dCas9-CDA-UL & pSC101, bla, dcas9-PmCDA1-UGI-LVA & 4 \\
\hline pgRNA01 & 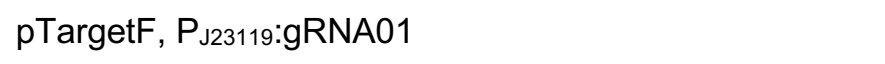 & This study \\
\hline pgRNA02 & pgRNA01, PJ23119:gRNA02 & This study \\
\hline pgRNA05 & pgRNA01, PJ23119:gRNA05 & This study \\
\hline pgRNA06 & pgRNA01, PJ23119:gRNA06 & This study \\
\hline pgRNA07 & pgRNA01, PJ23119:gRNA07 & This study \\
\hline pgRNA10 & pgRNA01, PJ23119:gRNA10 & This study \\
\hline pgRNA11 & pgRNA01, PJ23119:gRNA11 & This study \\
\hline pgRNA13 & pgRNA01, PJ23119:gRNA13 & This study \\
\hline pgRNA14 & pgRNA01, PJ23119:gRNA14 & This study \\
\hline pgRNA15 & pgRNA01, PJ23119:gRNA15 & This study \\
\hline pgRNA19 & pgRNA01, PJ23119:gRNA19 & This study \\
\hline $\mathrm{pFX}$ & pMTLdSpCas9, dcas9 fused with PmCDA-UGI-LVA tag & This study \\
\hline pFX01 & $\mathrm{pFX}, \mathrm{P}_{\mathrm{J} 23119: \mathrm{gRNA} 01}$ & This study \\
\hline pFX02 & $\mathrm{pFX}, \mathrm{PJ}_{\mathrm{J} 23119: \mathrm{gRNA} 02}$ & This study \\
\hline pFX05 & pFX, PJ23119:gRNA05 & This study \\
\hline pFX06 & pFX, PJ23119:gRNA06 & This study \\
\hline pFX07 & pFX, PJ23119:gRNA07 & This study \\
\hline pFX10 & pFX, PJ23119:gRNA10 & This study \\
\hline pFX11 & pFX, PJ23119:gRNA11 & This study \\
\hline $\mathrm{pFX} 13$ & 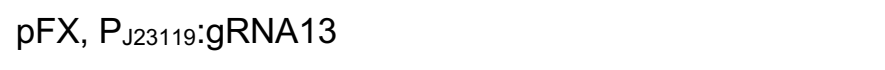 & This study \\
\hline pFX14 & 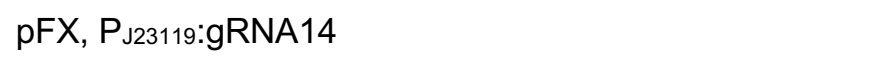 & This study \\
\hline pFX15 & 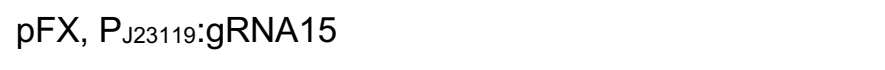 & This study \\
\hline pFX19 & pFX, PJ23119:gRNA19 & This study \\
\hline
\end{tabular}


Table S6 Primers used for plasmid construction

\begin{tabular}{|c|c|c|}
\hline Primer & Sequence & Note \\
\hline EBT-PFX-080 & GATTTGAGTCAGCTAGGAGGTGACGGTGGAGGAGGTTCTGGAGG & \multirow{6}{*}{$\begin{array}{l}\mathrm{pFX} \\
\text { series }\end{array}$} \\
\hline EBT-PFX-086 & ATGCCTGGAGATCCTTACTCGAGTTATGCAACCAGTCCTAGCATCTTG & \\
\hline EBT-PFX-081 & ССТCCAGAАССТССТССАССGТСАССТССТАGСТGАСТСАААTС & \\
\hline EBT-PFX-087 & CAAGATGCTAGGACTGGTTGCATAACTCGAGTAAGGATCTCCAGGCAT & \\
\hline EBT-PFX-088 & GGCTCACCTTCGGGTGGGCCTTTCTGCGTTACCGCATATGCTGGATCCTT & \\
\hline EBT-PFX-089 & $\begin{array}{l}\text { ACGTTGTAAAACGACGGCCAGTGCCGAGCTCTGCAGGTCGACTCTAGAG } \\
\text { AAT }\end{array}$ & \\
\hline EBT-PFX-090 & TTACCTTTTCATTCCCTACAGTTTTAGAGCTAGAAATAGC & \multirow{2}{*}{ gRNA01 } \\
\hline EBT-PFX-091 & TGTAGGGAATGAAAAGGTAAGCTAGCATTATACCTAGGAC & \\
\hline EBT-PFX-133 & AAAAATTTGGAGTAAGGCAAGTTTTAGAGCTAGAAATAGC & \multirow{2}{*}{ gRNA02 } \\
\hline EBT-PFX-134 & TTGCCTTACTCCAAATTTTTTGCTAGCATTATACCTAGGAC & \\
\hline EBT-PFX-139 & CAAATAGTAAAGACAGCTCCGTTTTAGAGCTAGAAATAGC & \multirow{2}{*}{ gRNA05 } \\
\hline EBT-PFX-140 & GGAGCTGTCTTTACTATTTGGCTAGCATTATACCTAGGAC & \\
\hline EBT-PFX-141 & AATTGATCACTATCTGGGCAGTTTTAGAGCTAGAAATAGC & \multirow{2}{*}{ gRNA06 } \\
\hline EBT-PFX-142 & TGCCCAGATAGTGATCAATTGCTAGCATTATACCTAGGAC & \\
\hline EBT-PFX-143 & TGAAATTGATGGAAAAAATTGTTTTAGAGCTAGAAATAGC & \multirow{2}{*}{ gRNA07 } \\
\hline EBT-PFX-144 & AАTTTTTTTCCATCAATTTCAGCTAGCATTATACCTAGGAC & \\
\hline EBT-PFX-153 & ATCCATCCTATAATTCCTTCGTTTTAAGAGCTAGAAATAGC & \multirow{2}{*}{ gRNA10 } \\
\hline EBT-PFX-154 & GAAGGAATTATAGGATGGATGCTAGCATTATACCTAGGAC & \\
\hline EBT-PFX-155 & AACAAGTGGATGAAATTTTCGTTTTAGAGCTAGAAATAGC & \multirow{2}{*}{ gRNA11 } \\
\hline EBT-PFX-156 & GAAAATTTCATCCACTTGTTGCTAGCATTATACCTAGGAC & \\
\hline EBT-PFX-178 & GGTCAGGGAATGCCAACTTAGTTTTAGAGCTAGAAATAGC & \multirow{2}{*}{ gRNA13 } \\
\hline EBT-PFX-179 & TAAGTTGGCATTCCCTGACCGCTAGCATTATACCTAGGAC & \\
\hline EBT-PFX-180 & GATCAAGCAGATAAGATCAGGTTTTAGAGCTAGAAATAGC & \multirow{2}{*}{ gRNA14 } \\
\hline EBT-PFX-181 & CTGATCTTATCTGCTTGATCGCTAGCATTATACCTAGGAC & \\
\hline EBT-PFX-182 & TTCAAATAGTAAAGACAGCTCCGTTTTAGAGCTAGAAATAGC & \multirow{2}{*}{ gRNA15 } \\
\hline EBT-PFX-183 & GGAGCTGTCTTTACTATTTGAAGCTAGCATTATACCTAGGAC & \\
\hline EBT-PFX-201 & AATCAAGCAGATAAAATAAGGTTTTAGAGCTAGAAATAGC & \multirow{2}{*}{ gRNA19 } \\
\hline EBT-PFX-202 & CTTATTTTATCTGCTTGATTGCTAGCATTATACCTAGGAC & \\
\hline
\end{tabular}


Table S7 Primers used for the verification of base editing

\begin{tabular}{lll}
\hline Primer & Sequence & Note \\
\hline EBT-PFX-131 & AGGATAGGACATACCCTGTG & Verification of pta \\
EBT-PFX-132 & CATCTACAGACATGCCTGTTC & editing \\
EBT-PFX-130 & GGAGTAAGGCAAAGGAAGAC & Sequencing for pta \\
EBT-PFX-166 & TCCCCAATTTAGCATACTAGGC & Verification of adhE1 \\
EBT-PFX-167 & CACATATGCCTCCAGTGCAT & editing \\
EBT-PFX-168 & TTACTGACTGCTCTGAGGCA & Sequencing for adhE1 \\
EBT-PFX-169 & ATGCACTGGAGGCATATGTG & Verification of adhE2 \\
EBT-PFX-170 & GTGCAACTCCAAGACTACCAT & editing \\
EBT-PFX-171 & AGGAGCACCAGCTTTAACTG & Sequencing for adhE2 \\
EBT-PFX-163 & TGAAGAAGCGCTTCAAGTTC & Verification of aor1 \\
EBT-PFX-164 & CTGCCTCTAATAGTGAATCTGC & editing \\
EBT-PFX-200 & CGTTGGTGCAGTTATGGGAT & Sequencing for aor1 \\
EBT-PFX-187 & CTAAGGCAATGGGGATTGGA & Verification of aor2 \\
EBT-PFX-188 & AGTTTCCACCTCCTTAGGCTA & editing \\
EBT-PFX-189 & GGGAGCAGAATCAAAGCAG & Sequencing for aor2 \\
\hline
\end{tabular}




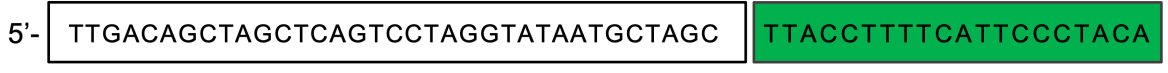

GTTTTAGAGCTAGAAATAGCAAGTTAAAATAAGGCTAGTCCGTTATCAACTTGAAAAA

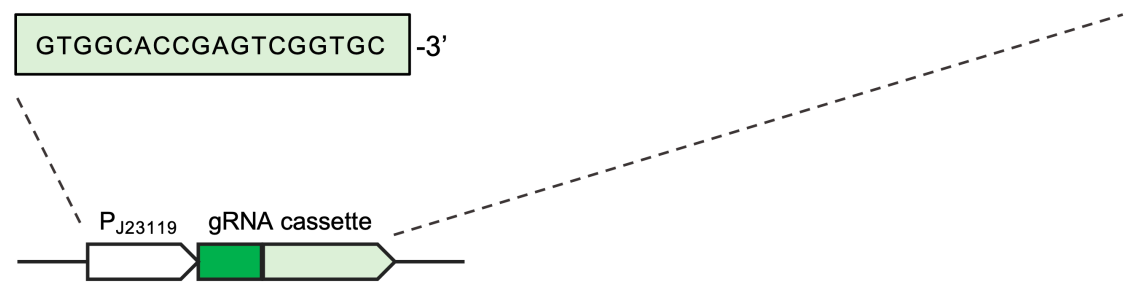

Targeting module

Figure S1. Scheme and sequence of gRNA cassette. Taking gRNA01 as an example, the sequences of $\mathrm{P}_{\mathrm{J} 23119}$ promoter (white), gRNA01 (green), and gRNA scaffold of dCas9 from S. pyogenes (light green) are shown. For each line, the sequence reads from left to right and does not indicate the structure. Both strands of the target sequence are displayed. The gRNA01 will bind the complimentary strand of the editing strand, and potentially edits the Cs on the editing strand within the editing window. The nucleotide directly adjacent to the protospacer adjacent motifs (PAM) is counted as position -1 and the starting position. 


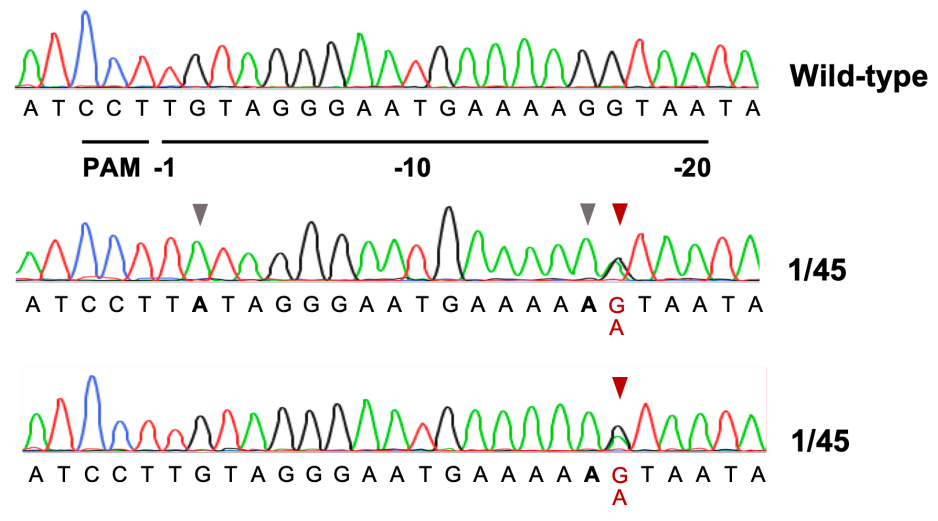

Figure S2. Mixed signals in the two colonies when editing pta using gRNA01. Grey arrows indicate clean mutations, and red arrows indicate mixed signals of $G$ and $A$. (PAM: protospacer adjacent motifs.) 
A

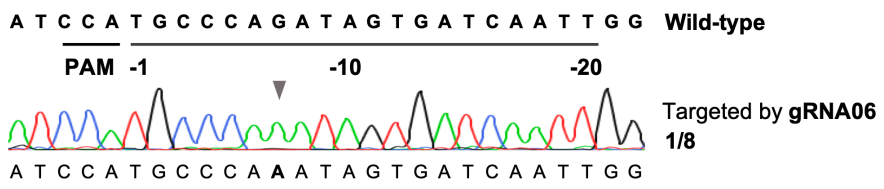

B

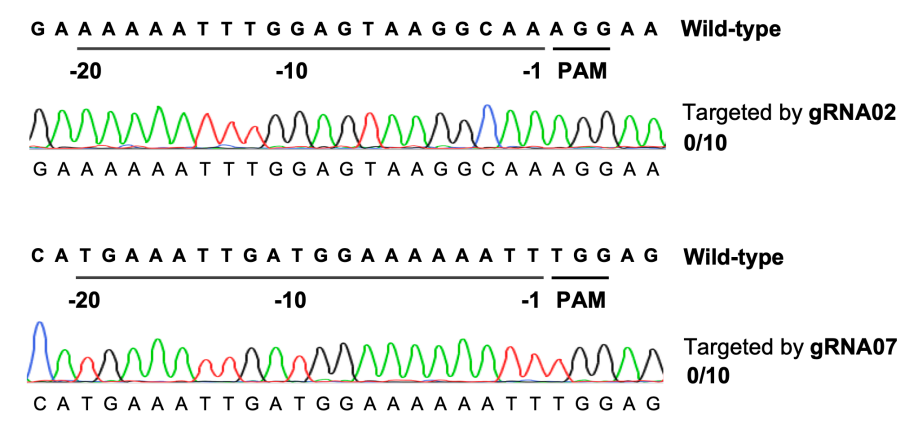

C

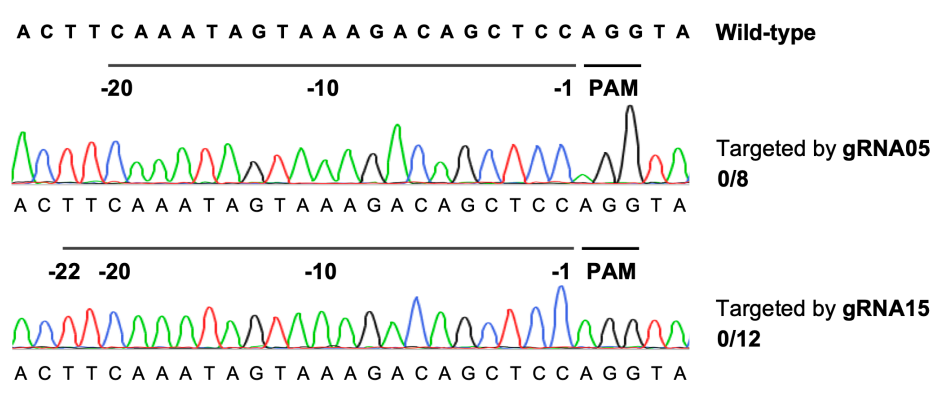

Figure S3. Editing principles. (A) Base editing in pta with gRNA06. Grey arrow indicates successful editing. (B) Evaluation of base editing on target sequences without Cs using gRNA02 and gRNA07. (C) Base editing with different length of gRNAs (gRNA05 and gRNA15) for editing the $\mathrm{C}$ at position -20. (PAM: protospacer adjacent motifs.) 
QX3 (10 transfers/ca. 66.2 generations)

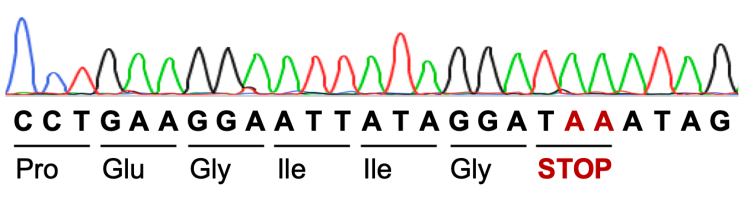

QX4 (10 transfers/ca. 66.1 generations)

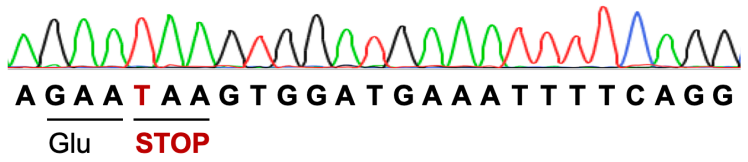

QX5 (10 transfers/ca. 65.9 generations)

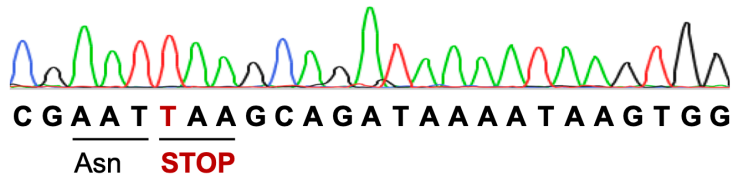

QX6 (10 transfers/ca. 66.5 generations)

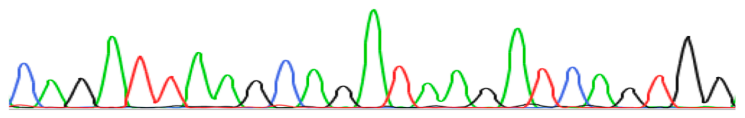

C A G A T T A A G C A G A T A A G A T C A G T G G Asn $\overline{\text { STOP }}$

Figure S4. Stability test of four edited strains. QX3, QX4, QX5, and QX6 were transferred 10 times in RCM and the single-nucleotide variations were sequenced to check stability. The sequencing results of the 10th transfer are shown here. 
A

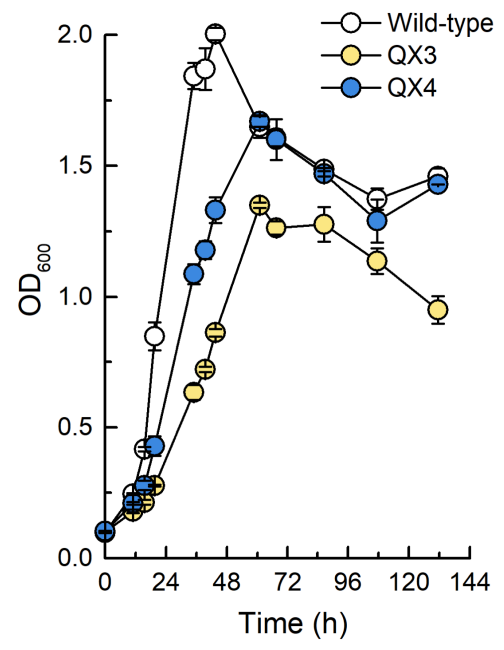

C

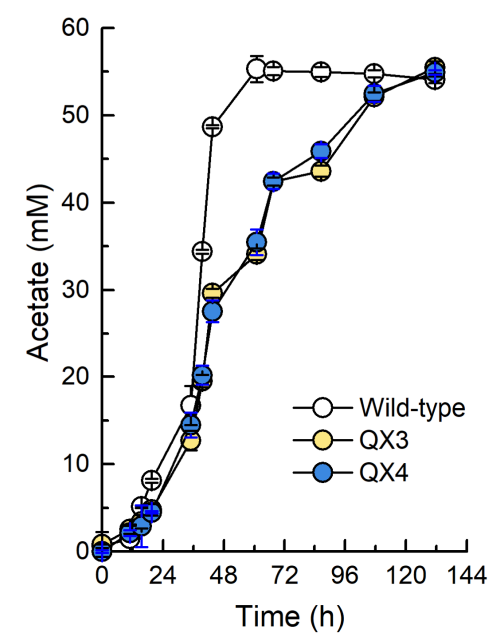

B

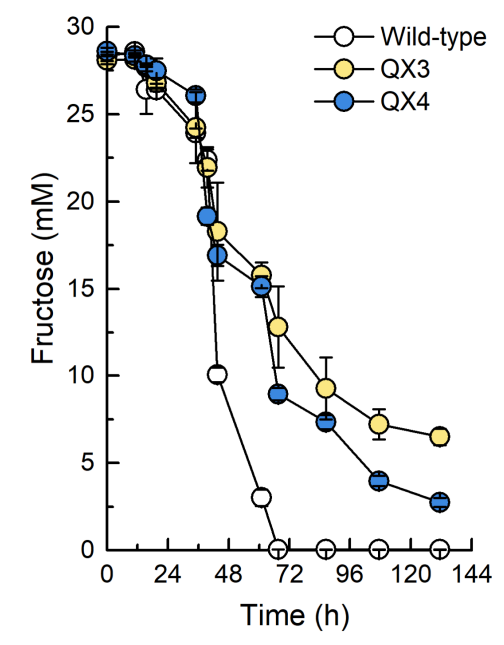

D

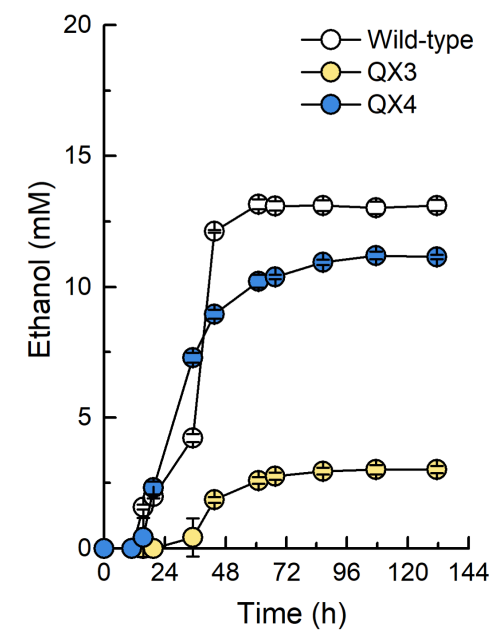

Figure S5. Fermentation performances of wild-type, QX3 (adhE1 Trp169*), and QX4 (adhE2 Gln33*) under heterotrophic conditions with $5 \mathrm{~g} / \mathrm{L}(27.8 \mathrm{mM})$ of fructose as the carbon source. (A) Growth, (B) concentration of fructose, (C) concentrations of acetate, and (D) concentration of ethanol in the culture during fermentation. 
A

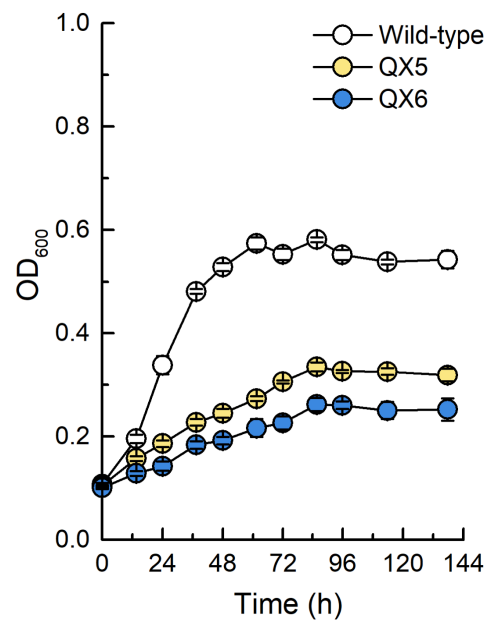

C

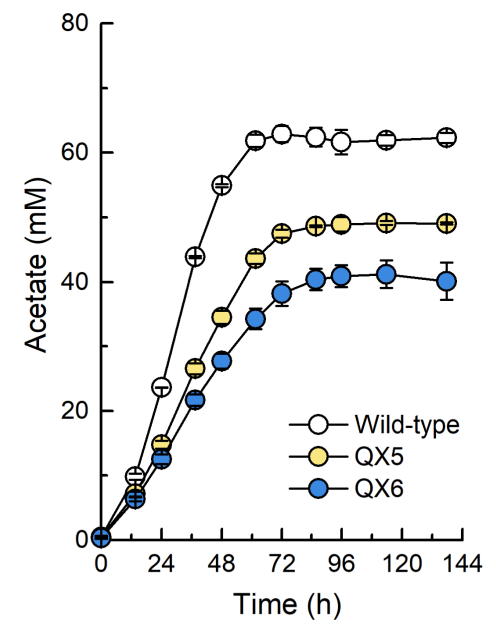

B

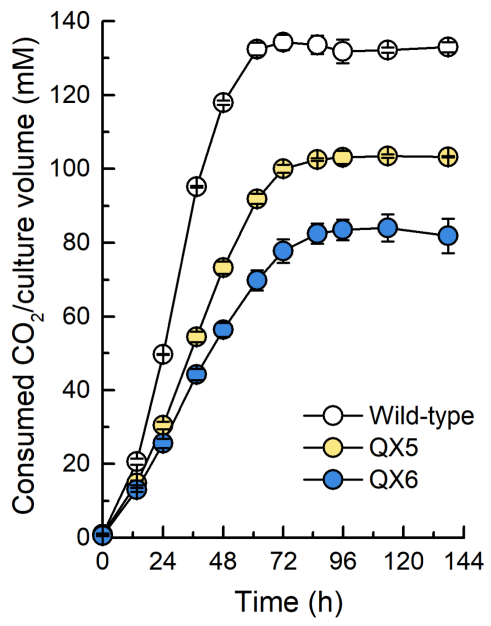

D

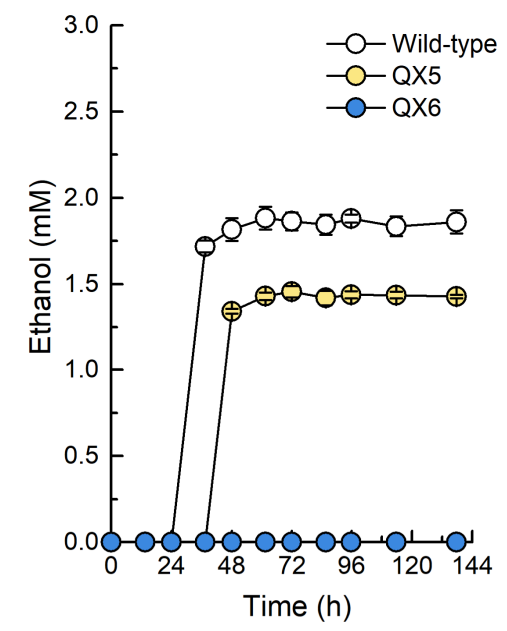

Figure S6. Fermentation performance of wild-type, QX5 (aor1 GIn267*), and QX6 (aor2 GIn267*) under autotrophic conditions with a gas mixture $\left(\mathrm{H}_{2} / \mathrm{CO}_{2}, 80 / 20\right.$ vol-\%, 1.5 bar $)$ as the substrate. (A) Growth, $(\mathrm{B})$ consumed $\mathrm{CO}_{2}$ per culture volume, $(\mathrm{C})$ concentrations of acetate, and (D) concentration of ethanol in the culture during fermentation. 

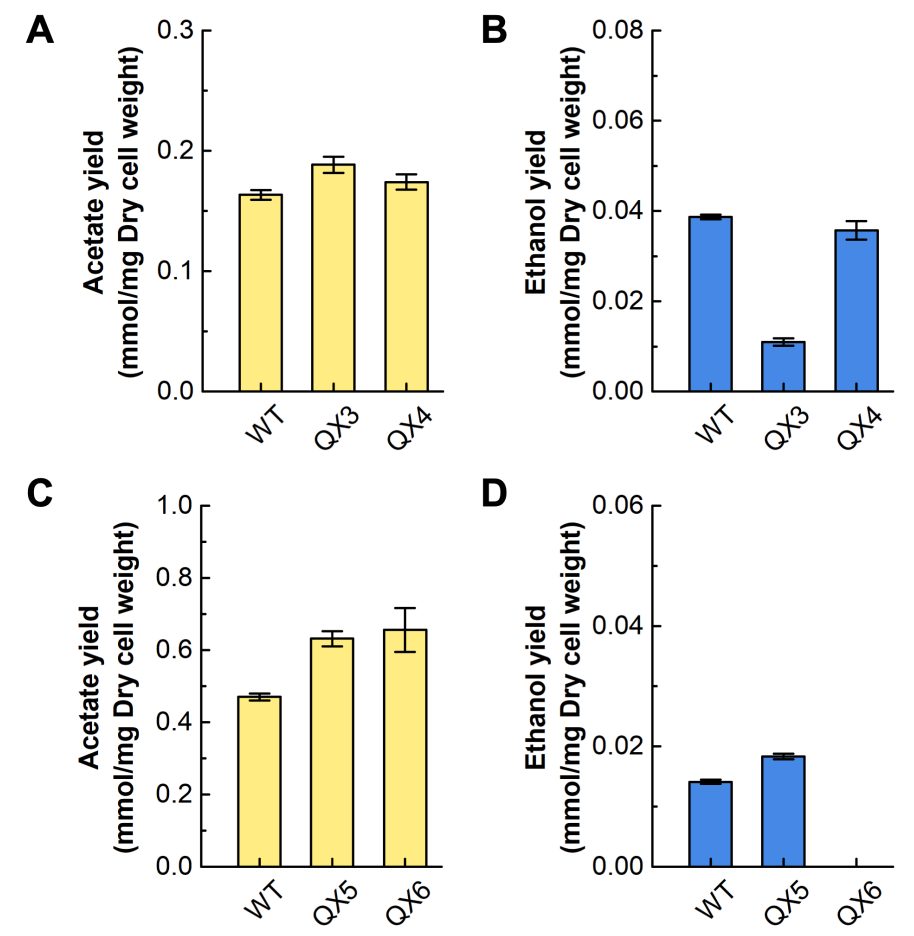

Figure S7. Acetate and ethanol yields per dry cell weight. Acetate $(A)$ and ethanol $(B)$ yields of wild-type, QX3, and QX4 under heterotrophic conditions with $5 \mathrm{~g} / \mathrm{L}(27.8 \mathrm{mM})$ of fructose as the carbon source. Acetate (C) and ethanol (D) yields of wild-type, QX5, and QX6 under autotrophic conditions with a gas mixture of $\mathrm{H}_{2} / \mathrm{CO}_{2}(80 / 20$ vol- $\%, 1.5$ bar) as the substrate. The fermentation experiments were conducted in triplicate $(\mathrm{N}=3)$, and the error bars indicate the standard deviations. 


\section{References}

1. Molitor, B., Kirchner, K., Henrich, A. W., Schmitz, S., and Rosenbaum, M. A. (2016) Expanding the molecular toolkit for the homoacetogen Clostridium ljungdahlii. Sci. Rep. 6, 31518.

2. Woolston, B. M., Emerson, D. F., Currie, D. H., and Stephanopoulos, G. (2018) Rediverting carbon flux in Clostridium ljungdahlii using CRISPR interference (CRISPRi). Metab. Eng. 48, 243-253.

3. Jiang, Y., Chen, B., Duan, C., Sun, B., Yang, J., and Yang, S. (2015) Multigene editing in the Escherichia coli genome via the CRISPR-Cas9 system. Appl. Environ. Microbiol. 81, 2506-14.

4. Banno, S., Nishida, K., Arazoe, T., Mitsunobu, H., and Kondo, A. (2018) Deaminasemediated multiplex genome editing in Escherichia coli. Nat. Microbiol. 3, 423-429. 